\section{Identification of the High Frequency of Triploid Potato Resulting from Tetraploid $\times$ Diploid Crosses}

\author{
Ryan C. Graebner ${ }^{1}$ \\ Columbia Basin Agricultural Research Center, Oregon State University, \\ Adams, OR 97810 \\ Hsuan Chen $^{2}$ and Ryan N. Contreras
Department of Horticulture, Oregon State University, Corvallis, OR 97331
}

Kathleen G. Haynes ${ }^{4}$

USDA-ARS, Genetic Improvement of Fruits and Vegetables Laboratory, Beltsville, MD 20705

Vidyasagar Sathuvalli,
Hermiston Agricultural Research \& Extension Center, Hermiston, OR 97838

Additional index words. Ploidy, unreduced gametes, triploid block, flow cytometry, chromosome count

\begin{abstract}
Conventional wisdom regarding potato breeding indicates that a strong triploid block prevents the development of viable triploid seeds from crosses between tetraploid and diploid clones. However, in a recent set of crosses between elite tetraploid potatoes and an improved diploid hybrid population derived from group Stenotomum and group Phureja, $61.5 \%$ of the resulting clones were found to be triploid. If clones derived from one diploid parent suspected of producing a high frequency of unreduced gametes were excluded, then the frequency of triploid clones increased to $74.4 \%$. Tubers of these triploids are generally intermediates of the two parental groups. Our findings indicate the possibility of using triploid potatoes in potato variety development programs and in genetic and genomic studies.
\end{abstract}

The term "triploid block" was first used by Marks (1966a) to describe the observation that "...although triploids do occur in the majority of suitable crosses, their frequency is often far below expectation..." Although this study specifically focused on several sets of crosses between clones of Solanum chacoense that were expected to yield a high proportion of triploid clones, the conclusion reflected previous observations that tetraploid $\times$ diploid crosses often result in a low seed set. This observation is consistent with the relative scarcity of triploid landrace cultivars in South America, as described by Jackson et al. (1977). To help explain these and other disparities between the expected and realized ploidy frequencies, Johnston et al. (1980) proposed the endosperm balance number (EBN) hypothesis, which states that for successful endosperm formation to occur, the effective ploidy, determined by EBNs, must have a 2 maternal : 1 paternal ratio. For

\footnotetext{
Received for publication 5 Dec. 2018. Accepted for publication 1 Apr. 2019.

${ }^{1}$ Assistant Professor and Former Graduate Research Assistant.

${ }^{2}$ Graduate Student.

${ }^{3}$ Associate Professor.

${ }^{4}$ Research Geneticist.

${ }^{5}$ Corresponding author. E-mail: vidyasagar@ oregonstate.edu.
} presumably because these clones produced an increased frequency of unreduced gametes. Van Suchtelen (1976) produced a low frequency of triploids and concluded that triploid clones generally resembled their tetraploid siblings in terms of morphology and yield. Finally, De Maine (1994) found that tetraploid group Tuberosum $\times$ diploid group Phureja crosses could result in $8 \%$ to
$71 \%$ triploid plants, depending on the tetraploid parent. Despite the moderate to high frequency of triploid plants in these crosses, overall triploid production was consistently low, with plants rarely producing more than a few triploid seeds per fruit.

In addition to crosses in which both parents were from $S$. tuberosum, Johnston and Hanneman (1995) found that some group Andigena clones produced a relatively high number of triploid seeds when pollinated with $S$. chacoense clones, with an average of 17 triploids per fruit for one group Andigena clone. However, the authors concluded that this trait had low heritability, which could complicate efforts to replicate this high triploid yield in crosses relevant to variety development efforts.

Marks (1966b) noted the following paradox: although triploid clones are difficult to produce and would therefore constitute a very small proportion of the naturally occurring seeds in cultivated potatoes, triploid clones are relatively abundant in South American landraces. Furthermore, it was suggested that for this situation to occur, triploids must have some selective advantage over other ploidy levels (Marks, 1966b).

For other crops, triploids are best known for causing seedless fruit, such as banana, watermelon, and citrus. However, few traits specifically attributable to triploidy have been reported in the literature. Many commercial European cultivars of sugar beet are triploid hybrids (Sliwinska and Lukaszewska, 2005), which is mainly because triploid sugar beets had higher root yields than diploid hybrids (Peto and Boyes, 1940). However, the reason for this production advantage is not clear (Sliwinska and Lukaszewska, 2005).

Potato breeding programs worldwide perform various tetraploid $\times$ diploid crosses for effective introgression of biotic and abiotic stress resistance traits from diploid to tetraploid potatoes using unreduced gametes. In an effort to study and quantify the performance of crosses between diploid and tetraploid potato clones for future introgression, we performed a series of crosses using advanced diploid clones and elite tetraploid breeding material. To confirm a successful cross and ploidy, we conducted flow cytometry to remove any diploids because they would have been irrelevant to that study. Here, we report the identification of a high frequency of triploids from tetraploid $x$ diploid crosses that were confirmed through somatic chromosome counts and flow cytometry.

\section{Materials and Methods}

Plant material. Twelve diploid clones were selected from the cycle four late blightresistant hybrid population derived from group Phureja and group Stenotomum clones (Haynes, 1972, 1980; Haynes et al., 2014). This diploid population was selected for tuberization under long day growing conditions, specific gravity, and late blight resistance over 
15 cycles of maternal half-sib selection. Eighteen elite tetraploid clones from group Tuberosum were selected from clones used in the

Table 1. List of parents used in tetraploid $\times$ diploid crosses.

\begin{tabular}{lc}
\hline Clone name & Ploidy \\
\hline Snowden & $4 \mathrm{x}$ \\
Atlantic & $4 \mathrm{x}$ \\
EVA & $4 \mathrm{x}$ \\
Lamoka & $4 \mathrm{x}$ \\
Ivory Crisp & $4 \mathrm{x}$ \\
A00710-1VR & $4 \mathrm{x}$ \\
AO03123-2 & $4 \mathrm{x}$ \\
OR01007-3 PVY & $4 \mathrm{x}$ \\
ORAYT-9 (PVY) & $4 \mathrm{x}$ \\
Castle Russet & $4 \mathrm{x}$ \\
Payette Russet & $4 \mathrm{x}$ \\
A06866-2PVY adg & $4 \mathrm{x}$ \\
A07547-4VR & $4 \mathrm{x}$ \\
A08640-2PCN & $4 \mathrm{x}$ \\
PALB03016-3 & $4 \mathrm{x}$ \\
TACNA & $4 \mathrm{x}$ \\
BD1202-2 & $2 \mathrm{x}$ \\
BD1205-4 & $2 \mathrm{x}$ \\
BD1216-3 & $2 \mathrm{x}$ \\
BD1222-1 & $2 \mathrm{x}$ \\
BD1240-6 & $2 \mathrm{x}$ \\
BD1244-1 & $2 \mathrm{x}$ \\
BD1244-3 & $2 \mathrm{x}$ \\
BD1247-3 & $2 \mathrm{x}$ \\
BD1251-1 & $2 \mathrm{x}$ \\
BD1253-4 & $2 \mathrm{x}$ \\
BD1257-5 & $2 \mathrm{x}$ \\
BD1268-1 & $2 \mathrm{x}$ \\
BD1269-1 & $2 \mathrm{x}$ \\
\hline &
\end{tabular}

Oregon State University potato breeding program (Table 1). Tetraploid clones were selected on the basis of superior agronomic traits. In addition to the diploid and tetraploid potatoes, the clones PI 595441 from Solanum juzepczukii and PI 604206 from Solanum curtilobum were included as examples of triploid and pentaploid potatoes, respectively.

Crossing. Plants were grown in a greenhouse in 19-L containers filled with LA4PC (Sun Gro Horticulture H, Agawam, MA) potting soil amended with $1 \mathrm{~g} / \mathrm{L}$ 15-9-12 Osmocote Smart-Release Plant Food Plus Outdoor and Indoor formulation (The Scotts Company, Marysville, OH). After planting, the plants were irrigated with water supplemented with Jack's Classic No. 4 20-20-20 fertilizer (J.R. Peters Inc., Allentown, PA) at a rate of $200 \mathrm{ppm}$ as needed. The greenhouse temperature was set to a daytime temperature of $24^{\circ} \mathrm{C}$ and a nighttime temperature of $20{ }^{\circ} \mathrm{C}$, and natural light was supplemented with a combination of metal halide and high-pressure sodium lights during a 20hour photoperiod. As plants grew, all but one or two shoots were snapped off. Occasionally, when a main shoot appeared to be losing vigor, the main shoot was snapped off at the tip, and a lateral shoot was allowed to restore vigor. Plants were staked with bamboo sticks.

Approximately 200 pollinations were attempted, and all used the tetraploid clone as the female parent and the diploid clone as the male parent. Specific combinations of

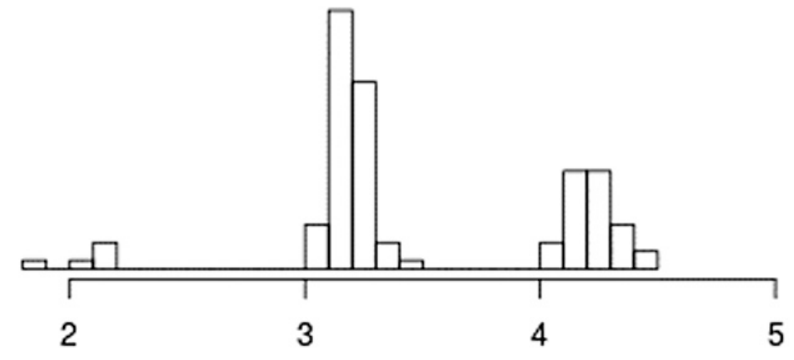

Genome Size (pg)

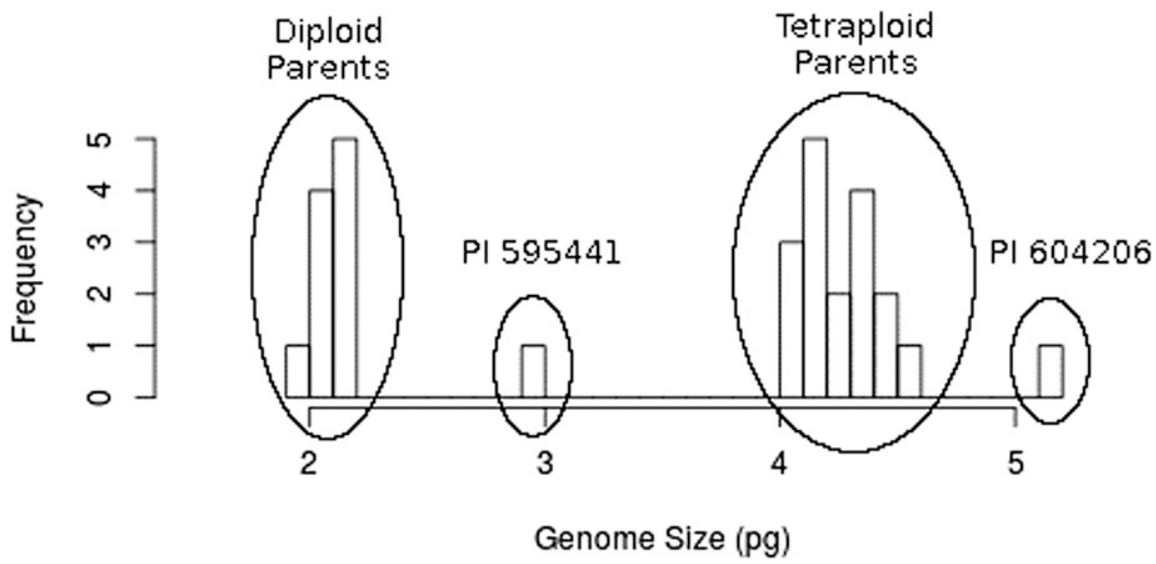

Fig. 1. (A) Estimated genome weights of 96 clones resulting from tetraploid $\times$ diploid crosses. (B) Estimated genome size of parents of tetraploid $\times$ diploid crosses, the triploid clone PI 595441 from $S$. juzepczukii, and the pentaploid clone PI 604206 from S. curtilobum. tetraploid and diploid parents that were crossed were based on pollen and receptive stigma availability at the time of crossing. In general, we attempted to make as many unique crosses as possible.

During pollen collection, anthers were removed from flowers at anthesis, as determined by a black spot at the tip of each anther, placed in parchment paper envelopes, and left in the greenhouse for $\approx 24 \mathrm{~h}$. Then, each closed envelope was vibrated using an electric palm sander without sand paper attached. Next, the envelope was opened and pollen was collected using a knife. Pollen was stored in plastic serum vials in the refrigerator for up to 1 month.

To enable pollination, the petals of unopened flowers were gently opened with tweezers, and the flowers were emasculated. Small glassine bags were stapled over each flower before pollination. After 1 to $2 \mathrm{~d}$, one edge of the glassine bag was cut with scissors, and a very small metal spatula was used to coat the stigma with pollen. After pollination, the cut edges of the glassine bags were stapled and monitored for hybridization success.

Development of triploids. Fruits were collected when they could be easily broken from the plants. After fruits became soft $(\approx 30$ d), they were slit open with a scalpel. Then, seeds were carefully removed and placed on a paper towel to dry. All fruits obtained from tetraploid $\times$ diploid crosses had very low seed sets. Most fruits had fewer than five seeds, and no single fruit had more than 20 seeds. Seeds were typically found embedded in portions of the placenta that were fleshier than the surrounding tissue. When the seeds were dry, they were stored in paper envelopes until germination.

Seeds were placed in plastic serum vials with $0.1 \%$ gibberellic acid for $\approx 24 \mathrm{~h}$. Then, seeds were placed on damp paper towels in petri dishes, which were then placed in opaque, humid, plastic tote boxes in the greenhouse. The seeds were monitored multiple times per day and moistened with water from a spray bottle as needed. As the cotyledons emerged from the seeds $(\approx 7 \mathrm{~d})$, seedlings were transferred to trays of $2.5-\mathrm{cm}$ pots filled with Sun Gro LA4PC potting mix (Sun Gro Horticulture $\mathrm{H}$ ). Irrigation water was amended with Jack's Classic No. 4 20-2020 fertilizer (J.R. Peters Inc.) at a rate of $200 \mathrm{ppm}$.

After 3 weeks, seedlings were transferred to 2-L pots filled with Greenhouse Mix \#3, (Teufel Products Co., Hillsboro, OR) amended with $2 \mathrm{~g} / \mathrm{L}$ 15-9-12 Osmocote smart-release plant food plus outdoor \& indoor formulation (The Scotts Company). Approximately $75 \mathrm{~d}$ after seedlings were transferred to 2-L pots, mini-tubers were collected from each pot and stored for later use.

Tuber observation. When enough seedling tubers were produced in the $2-\mathrm{L}$ pots, clones resulting from tetraploid $\times$ diploid crosses were first planted in four-hill plots in Klamath Falls, OR, and four-hill plots in Hermiston, OR depending on 
seedling tuber availability, in 2017. Most of the parents were planted in both locations. Clones BD1205-4, BD1244-3, and BD12691 were discarded immediately after crossing due to potato virus $\mathrm{Y}$ infection. Plots were grown using standard agricultural practices for their respective regions. At the end of the growing season, the tubers were harvested and checked for tuberization.

Flow cytometry. Fresh leaf tissue samples of each clone derived from tetraploid $x$ diploid crosses, the parents, and the clones PI 595441 and PI 604206 were collected from pots in the greenhouse or from plants in the field and used for ploidy analysis. Flow cytometry was conducted using either a CyFlow Ploidy Analyser (Sysmex Corporation, Kobe, Japan) or a CyFlow Space flow cytometer system (Sysmex Partec GmbH, Görlitz Germany) with CyStain ultraviolet Precise P (Sysmex Corporation). Five triploid clones were measured with both methods to confirm that the different methods did not provide substantially different results. Relative fluorescence of Pisum sativum variety 'Ctirad' (8.76 pg/2C) (Lattier and Contreras, 2017) was used as a standard to determine the genome size of potato samples.

Somatic chromosome counts. Tubers from three selected triploid clones (based on flow cytometry results), three diploid parents, one tetraploid parent, and clone 595441 from $S$. juzepczukii were planted in $2-\mathrm{L}$ pots in the greenhouse. After 1 to 2 weeks, 5 to 10 quickly growing root tips were collected from each clone at $\approx 2: 00 \mathrm{PM}$ and placed in $2 \mathrm{~mm}$ hydroxyquinoline for $3 \mathrm{~h}$ in the light at room temperature. Then, root tips were rinsed in distilled water. Next, they were fixed in a solution of $75 \%$ ethanol and $25 \%$ acetic acid for storage that lasted up to several months at $4{ }^{\circ} \mathrm{C}$.

Root tips were treated with the enzyme solution described by Lattier et al. (2017) for
$1 \mathrm{~h}$ in an incubator set to $37{ }^{\circ} \mathrm{C}$. After the enzyme treatment, roots were transferred to a new slide using a pipette. One to two drops of modified Farmer's fixative (3 parts methanol : 1 part glacial acetic acid) were added to the root tip; then, root tip cells were separated by tapping the root tip with a metal spatula (Chen et al., 2015). A drop of modified Farmer's solution was added to each corner of the slide, and the solution was immediately lit with a match. Excess liquid was tapped off the slides, and the slides were allowed to air-dry overnight at $37{ }^{\circ} \mathrm{C}$. Airdried slides were submerged in a $5.7 \%$ solution of Giemsa stain modified solution (Sigma-Aldrich) for $15 \mathrm{~min}$, quickly rinsed in water, and air-dried overnight at $37{ }^{\circ} \mathrm{C}$. Images were obtained by a light microscope at $\times 200$ magnification (Axio Imager A1; Zeiss).

\section{Results}

Flow cytometry. The c-values were obtained from flow cytometry of the 96 clones obtained from tetraploid $\times$ diploid crosses clustered into three peaks corresponding to the diploid, triploid, and tetraploid levels, thereby enabling ploidy values to be assigned to each clone (Fig. 1). Of these clones, $5(5.2 \%)$ were diploid, $59(61.5 \%)$ were triploid, and $32(33.5 \%)$ were tetraploid (Table 2).

Seventeen of the 32 tetraploid clones shared a single diploid parent, BD1205-4. Only one triploid offspring was obtained from this parent. BD1205-4 tended to result in fruits with 5 to 20 seeds, as opposed to 1 to 4 seeds per fruit typical of other tetraploid $x$ diploid crosses. Our results suggested that BD1205-4 produced a high frequency of unreduced gametes, although this cannot be confirmed because we did not successfully maintain BD1205-4 after crossing. If clones with BD1205-4 as the male parent were excluded from this analysis, then $74.4 \%$ of the clones resulting from tetraploid $\times$ diploid crosses were triploid (Table 2).

Root squash. To confirm the ploidy determined with flow cytometry, root squashes for chromosome counts were carried on three diploid parents, a single tetraploid parent (cv. Eva), three triploid clones, and an additional triploid accession PI595441 from S. juzepczukii. Root squashes confirmed 24, 36, and 48 chromosomes for diploid, triploid, and tetraploid clones, respectively (Fig. 2). This showed that the ploidy values assigned by flow cytometry reflected the true ploidy values.

Tuber comparison. Each of the 58 triploid clones that were grown in Klamath Falls set tubers. Furthermore, 44 of the 46 triploid clones that were grown in Hermiston set tubers. The shapes and sizes of the tubers produced by the triploid potato clones were generally intermediates of the parents, suggesting that there are no consistent morphological characteristics of the whole-plant level specific to triploid potato clones. Examples of eight triploid clones with their parents are shown in Fig. 3.

\section{Discussion}

Although the high proportion of triploid clones obtained in the experiment is not in line with conventional wisdom, these results do share parallels with several previous works that have also reported triploids resulting from tetraploid $\times$ diploid crosses (Hanneman and Peloquin, 1968; De Maine, 1994; Van Suchtelen, 1976). In particular, Hanneman and Peloquin (1968) observed that for crosses with a higher seed set, the additional seeds were typically triploid, similar to what was observed from crosses using the diploid clone BD1205-4 as the male

Table 2. Frequency of ploidy levels from all tetraploid $\times$ diploid crosses and from tetraploid $\times$ diploid crosses that did not include the diploid clone BD1205-4.

\begin{tabular}{lrrr}
\hline & \multicolumn{3}{c}{ Progeny } \\
\cline { 2 - 4 } Cross & $2 \mathrm{x}$ & \multicolumn{3}{c}{$3 \mathrm{x}$} & $4 \mathrm{x}$ \\
\hline $\mathrm{x} \times 2 \mathrm{x}$ & $5(5.2 \%)$ & $59(61.5 \%)$ & $32(33.3 \%)$ \\
$4 \mathrm{x} \times 2 \mathrm{x}$ (Excluding BD1205-4) & $5(6.4 \%)$ & $8(74.4 \%)$ & $15(19.2 \%)$ \\
\hline
\end{tabular}

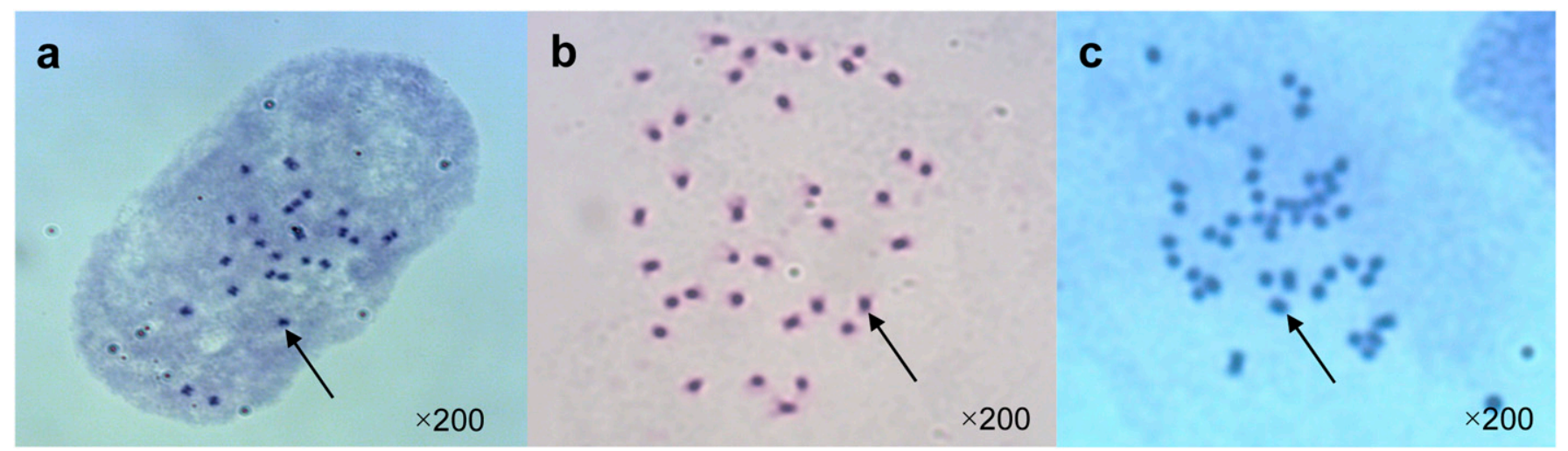

Fig. 2. Root squashes to confirm ploidy. (A) Diploid parent BD1222-1 $(2 n=2 x=24)$. (B) Triploid hybrid RP.2.3535 $(2 n=3 x=36)$. (C) Tetraploid parent cv. Eva $(2 n=4 x=48)$. The arrow points to a single chromosome. 


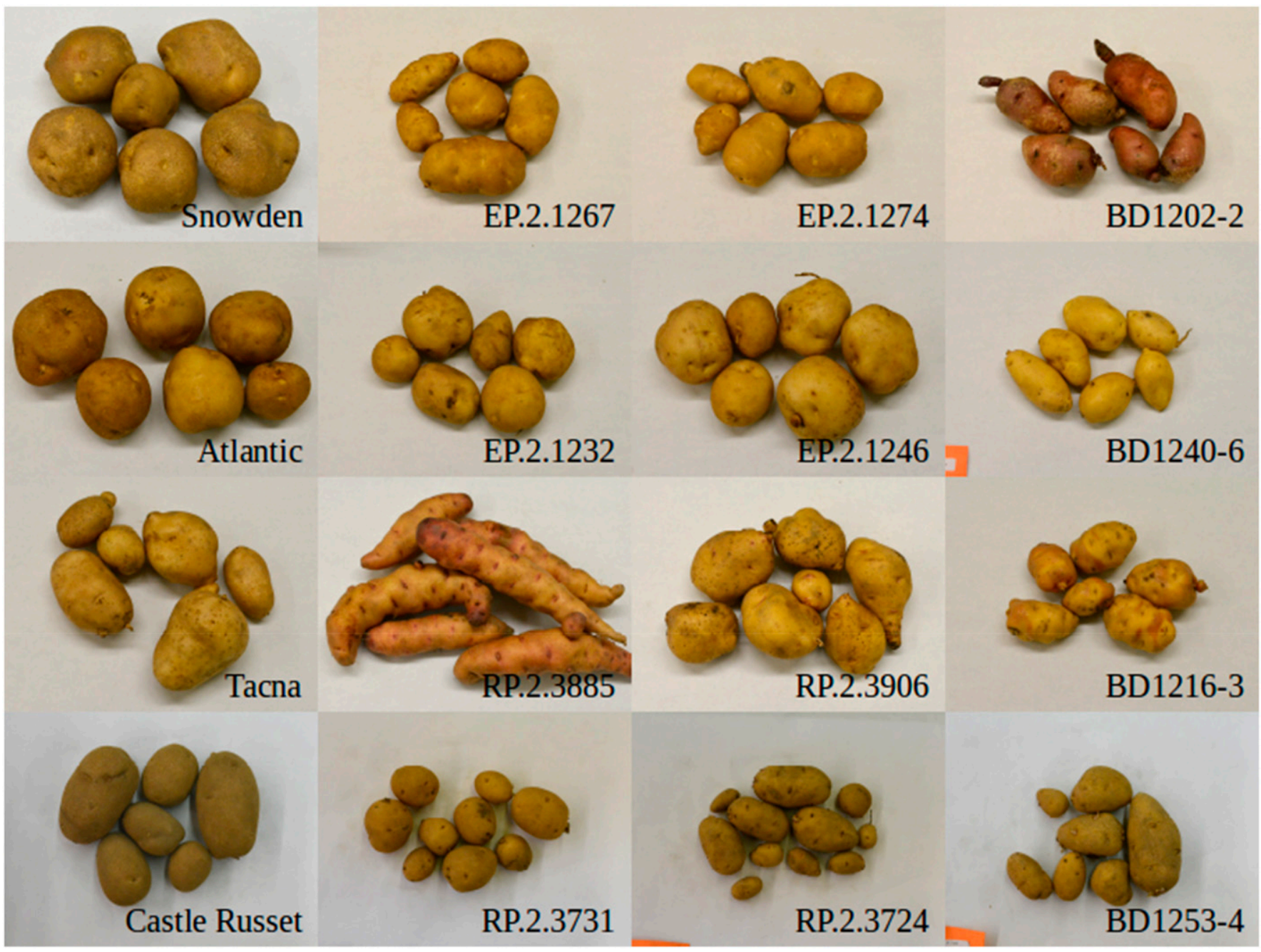

Fig. 3. Examples of triploids resulting from tetraploid $\times$ diploid crosses grown in Hermiston, OR. For each row, the clone on the left is the tetraploid parent, the clone on the right is the diploid parent, and the two clones in the center are triploid clones resulting from the cross between the two parents.

parent in this experiment. Furthermore, our results are in agreement with the observations made by Van Suchtelen (1976) that triploid clones generally resemble their tetraploid siblings.

Although the identification of triploid clones from tetraploid $\times$ diploid crosses is not entirely novel, the frequency of triploid clones relative to tetraploid clones observed in this experiment far exceeds that reported in previous studies. One possible explanation could be that genetic differences between the clones used in our study compared with those used in previous experiments either increased the likelihood of triploid formation through a reduction in the triploid block or decreased the likelihood of tetraploid formation through the decreased frequency of unreduced gametes in the male parent. Alternatively, the procedures we used to cross parents and germinate seeds in the experiment may have favored triploid production relative to other experiments. Much care was used in germination efforts, thereby allowing for the germination of seeds that appeared to have defects and, in a few cases, even the germination of seeds that appeared to have no endosperm.
Regarding variety development efforts, the identification of a relatively high frequency of triploids in our study partially reduced one of the barriers to developing triploid potato cultivars. However, due to the very low seed set, any triploid potato variety development efforts would require the investment of $\approx 100$-times the crossing effort to obtain a given number of seeds. Therefore, it would be necessary to demonstrate that triploid potato clones were superior to their diploid and tetraploid counterparts for such a triploid variety development program to be successful. Triploid potatoes are unlikely to serve as parents for germplasm improvement efforts because they are largely sterile, with some exceptions (Johnston and Hanneman, 1995; Magoon et al., 1962; Van Suchtelen, 1976). Nevertheless, our study showed that with increased crossing efforts and subsequent care of seedlings, it is possible to generate triploids from tetraploid $\times$ diploid potato crosses.

We also believe triploid potatoes may contribute to our understanding of the dosage effects of alleles for complex traits. With recent advances in high-throughput genome sequencing and chromosome sorting-based phased genome sequencing (Yang et al., 2011), the production of triploids could contribute to genomic studies and the development of haploid genome sequences and novel genomic regions contributed from the diploid parent. The use of triploids in genomic studies warrants future exploration.

\section{Literature Cited}

Chen, H., M.C. Chung, Y.C. Tsai, F.J. Wei, J.S. Hsieh, and Y.I.C. Hsing. 2015. Distribution of new satellites and simple sequence repeats in annual and perennial Glycine species. Bot. Stud. 56:22.

Hanneman, R.E. 1994. Assignment of endosperm balance numbers to the tuber-bearing Solanums and their close non-tuber bearing relatives. Euphytica 74:19-25.

Hanneman, R.E. and S.J. Peloquin. 1968. Ploidy levels of progeny from diploid-tetraploid crosses in the potato. Amer. Potato J. 45:255261.

Haynes, K.G., X. Qu, and B.J. Christ. 2014. Two cycles of recurrent maternal half-sib selection reduce foliar late blight in a diploid hybrid Solanum phureja - S. stenotomum population by two-thirds. Amer. J. Potato Res. 91:254259 
Haynes, F.L. 1972. The use of cultivated diploid Solanum species in potato breeding, p. 100 110. In: E.R. French (ed.). Prospects for the Potato in the Developing World. International Potato Center Symposium, Lima, Peru.

Haynes, F.L. 1980. Progress and future plans for the use of Phureja-Stenotomum populations, p. 80-88. In: OT Page (ed.). Utilization of the genetic Resources of the potato. III. Report of the Planning Conference. International Potato Center, Lima, Peru.

Jackson, M.T., J.G. Hawkes, and P.R. Rowe. 1977. The nature of Solanum $\times$ chaucha Juz. et Buk., a triploid cultivated potato of the South American Andes. Euphytica 26:775-783.

Johnston, S.A. and R.E. Hanneman, Jr. 1995. The genetics of triploid formation and its relationship to endosperm balance number in potato. Genome 38:60-67.
Johnston, S.A., T.P.M. den Nijs, S.J. Peloquin, and R.E. Hanneman. 1980. The significance of genic balance to endosperm development in interspecific crosses. Theor. Appl. Genet. 57:5-9.

Lattier, J.D. and R.N. Contreras. 2017. Ploidy and genome size in lilac species, cultivars, and interploid hybrids. J. Amer. Soc. Hort. Sci. 142:355-366

Lattier, J.D., H. Chen, and R.N. Contreras. 2017. Improved method of enzyme digestion for root tip cytology. HortScience 52:1029-1032.

Magoon, M.L., S. Ramanujam, and D.C. Cooper. 1962. Cytogenetical studies in relation to the origin and differentiation of species in the genus Solanum L. Caryologia 15:151-252.

De Maine, M.J. 1994. The ploidy composition of offspring from Solanum tuberosum $(4 \mathrm{x}) \mathrm{x} \mathrm{S}$. phureja $(2 \mathrm{x})$ crosses with special reference to triploid frequencies. Ann. Appl. Biol. 125:361366
Marks, G.E. 1966a. The origin and significance of intraspecific polyploidy: Experimental evidence from Solanum chacoense. Society for the Study of Evolution 20:552-557.

Marks, G.E. 1966b. The enigma of triploid potatoes. Euphytica 15:285-290.

Peto, F.H. and J.W. Boyes. 1940. Comparison of diploid and triploid sugar beets. Can. J. Res. 18:273-282.

Sliwinska, E. and E. Lukaszewska. 2005. Polysomaty in growing in vitro sugar-beet (Beta vulgaris L.) seedlings of different ploidy level. Plant Sci. 168:1067-1074.

Van Suchtelen, N. 1976. Triploids of the common potato, Solanum tuberosum L. Potato Res. 19:377-380.

Yang, H., X. Chen, and W.H. Wong. 2011. Completely phased genome sequencing through chromosome sorting. Proc. Natl. Acad. Sci. USA 108:12-17. 